\title{
Die Ziele langfristiger Geldanlage
}

\author{
Klaus Spremann; Universität St. Gallen
}

\section{$1 \quad$ Einleitende Übersicht}

Alle Privatinvestoren wollen ihr Geld "gut" anlegen und später einmal "ihr Geld mit Rendite zurückerhalten." Zur Modellierung dieser praktischen Aufgabe wird in der Regel ein Anlagehorizont als gegeben betrachtet und das Exposure in risikobehaftete Anlagen (Aktien) als Aufgabe formuliert, den Erwartungsnutzens des unsicheren Endvermögens zu maximieren. Ob die optimale Politik dynamisch ist oder ein konstantes Exposure vorsieht, hängt vom Typus der Risikonutzenfunktion ab. Empirische Untersuchungen sprechen für die Annahme konstanter relativer Risikoaversion und das Samuelson-Modell zeigt für diesen Fall, dass eine konstante Quote des (gesamten) Vermögens in Aktien investiert sein sollte. Außerdem erweist sich im Samuelson-Modell — der Intuition widersprechend - die Dauer der Geldanlage als irrelevant für die Aktienquote. Indessen wird in der Praxis immer wieder betont, dass das richtige Exposure hänge eben doch von der Länge des Anlagehorizonts und, was unbestritten ist, von der Höhe der Risikoaversion ab. Eine genauere Betrachtung der Rolle des sonstigen Vermögens zeigt, dass der Anlagehorizont doch Bedeutung hat, und zwar insoweit, als er die Höhe des sonstigen Vermögens beeinflußt. Eine genaue Erfassung der Dynamik des sonstigen Vermögens ist daher für die Ziel- und Strategieformulierung unerläßlich. Genauer kommt es nicht auf den Anlagehorizont direkt an, sondern nur auf die Höhe des sonstigen Vermögens zu jenem Zeitpunkt, zu dem der Investor sein "Geld mit Rendite zurückbekommen" möchte. Die Frage an den Investor, ob er, wenn der Zeitraum der Geldanlage beendet sein wird (wann immer das genau ist), noch über weiteres Vermögen verfügen können wird oder gar Verpflichtungen haben wird (negatives sonstiges Vermögen), ist am Ende wichtiger für die Anlagepolitik als die exakte Bestimmung der Risikoaversion. Um das Ergebnis der formalen Analyse anschaulich auszudrücken, lassen wir den Berater zum Privatinvestor sagen: "Ihre genaue Risikoaversion ist unwichtig, wichtig ist hingegen, ob sie ihre Geld einmal benötigen werden. Wenn Sie sich nur daran freuen, es zu haben, dann legen wir es zur Hälfte in Aktien an. Wenn Sie es jedoch irgendwann einmal benötigen - der Zeitpunkt ist unwichtig - legen wir das Geld sicher an: Es kommen nur Geldmarktinstrumente und Bonds in Frage."

Private Finanzinvestoren stehen ebenso wie institutionelle Investoren, die für private Anspruchsberechtigte handeln, regelmäßig vor der Aufgabe, eine optimal auf die individuellen Umstände und Präferenzen angepaßte Anlagestrategie für einen 
meist mehrjährigen Zeitraum zu bestimmen. ${ }^{1}$ Konkret geht es immer wieder um zwei Fragen, die das Exposure und die Selektion betreffen:

1. Welches Risikoexposure sollte die Person eingehen, und wie verändert sich das optimale Exposure im Zeitablauf? Welches Risiko ist folglich als Teil der langfristigen Politik gerade für das kommende Jahr einzugehen?

2. Welche Anlageinstrumente - Geldmarktinstrumente, Staatsanleihen, Corporate Bonds, Growth Stocks, Value Stocks, Small Caps, Optionen - sollten bis auf weiteres Bestandteil des Portfolios sein und in welcher relativen Gewichtung sollen sie in das Portfolio einbezogen werden?

Beide Aufgaben zusammen bilden den Kern der "Strategic Asset Allocation" und führen nach geeigneter Modellierung - insbesondere müssen die Ziele der langfristigen Geldanlage präzisiert werden — auf eine (stochastische) dynamische Optimierungsaufgabe. Deren Lösung kann durch die Bellmansche Gleichung (oder das Maximumprinzip von Pontrjagin) charakterisiert werden. ${ }^{2}$ Zur konkreten Berechnung der optimalen Politik im allgemeinen Fall müssen diese charakterisierenden Gleichungen diskretisiert und numerisch gelöst werden, was Approximationen verlangt. Die allgemeine Problemstellung ist daher recht komplex, selbst wenn die Zielsetzung klar ist und alle Parameter spezifiziert sind. ${ }^{3}$ Indessen sind in "Spezialfällen" einfachere Lösungen möglich.

Für den Fall einer Periode - es werden nur zu Beginn Entscheidungen getroffen, es gibt keine "zwischenzeitlichen" Veränderungen des Portfolios, und es gibt keine weitere Geldanlage nach Ende der einen Periode, die bereits jetzt antizipiert werden müßte - hat Tobin (1958) bewiesen, dass beide Aufgaben voneinander unabhängig gelöst werden können. Es ist heute ein Gemeingut: Die Lösung der zweiten Aufgabe besteht in der Bestimmung des Markt- oder Tangentialportfolios und die Lösung der ersten Aufgabe verlangt die Auswahl eines Portfolios auf der Capital Market Line entsprechend der Risikopräferenz des Investors.

Für den Mehrperiodenfall - der Investor kann das Portfolio "zwischenzeitlich" verändern ${ }^{4}$ - liegt die Möglichkeit der Separation hingegen nicht auf der Hand. Wir nennen Punkte, die eine getrennte und unabhängige Lösung der beiden Aufgaben verhindern. Wenn es Transaktionskosten für den Kauf und Verkauf der

1 Die "Lifetime Portfolio Selection" hat erstmals Samuelson (1969) aufgegriffen und später immer wieder Ergänzungen publiziert, so die Arbeit aus dem Jahre 1989, die das "Samuelson-Modell" darstellt. Zu umfassenden Untersuchungen der dynamischen und auf einen langen Zeithorizont bezogenen Portfolioselektion vergleiche Winhart (1999), Kränzlein (2000) sowie Campbell / Viceira (2002).

2 Luenberger (1979).

3 Eine Software hierzu bietet Prof. Gerd Infanger, University of Stanford, an.

4 Ungeachtet der Frage, ob die Veränderungsmöglichkeiten für das Portfolio nur diskrete Zeitpunkte sind oder ob dies "kontinuierlich" möglich sein soll. 
möglichen Anlageinstrumente gibt, ist jeder Kauf eines Wertpapiers zu einem Teil "irreversibel" und sollte daher nur getätigt werden, wenn auch noch übermorgen das Wertpapier Bestand des optimalen Portfolios sein sollte. Das hängt aber davon $\mathrm{ab}$, welches Exposure übermorgen noch gewünscht wird. Bei Transaktionskosten müssen daher die beiden Fragen simultan gelöst werden. Ähnliches gilt, wenn ein Zusammenhang zwischen dem gewünschten Exposure und der Menge möglicher Anlageinstrumente besteht, beispielsweise weil ein Investor mit geringer Risikotoleranz nur Geldmarktinstrumente, Staatsanleihen und vielleicht einige Value Stocks zuläßt, nicht dagegen Growth Stocks, Small Stocks oder Optionen. Schließlich lernen viele Investoren und können im Verlauf der Zeit aufgrund gemachter Erfahrungen die jeweiligen Risiken und Renditen der Anlageinstrumente besser einschätzen. Dieses Lernen ist jedoch daran geknüpft, dass die Instrumente tatsächlich genutzt werden, so dass auch bei Lernen die Aufgaben miteinander verknüpft sind und nicht unabhängig voneinander gelöst werden können. ${ }^{5} \mathrm{Zwei}-$ fellos ergibt sich eine wesentliche Vereinfachung der dynamischen PortfolioSelektion, wenn beide Aufgaben (Exposurebestimmung und Titelgewichtung) getrennt angegangen werden können - so wie Tobin das für den Einperiodenfall bewies. Dazu ist mithin Voraussetzung, dass es weder Transaktionskosten noch Zusammenhänge zwischen dem Risikoexposure beziehungsweise der Risikotoleranz und der Menge zulässiger Anlageinstrumente besteht, und auch die Dynamik des Lernens darf der separaten Behandlung nicht entgegenstehen. Ohne in eine Untersuchung einzusteigen, unter welchen (hinreichenden) Bedingungen bei der Strategischen Asset Allocation die Separation möglich ist, wollen wir beide Fragen als trennbar ansehen.

- Die Geldanlage wird dann zu einem jeden Zeitpunkt auf einer übergeordneten Ebene durch das Exposure gegenüber einer risikobehafteten Assetklasse beschrieben. Es geht hier also nur um das Sigma, die Volatilität, welche die augenblickliche Rendite des Portfolios der Person aufweisen sollte. Empirische Erkenntnisse und theoretische Modelle (Brownsche Bewegung) beschreiben einen Zusammenhang zwischen Sigma (Risiko) und Renditeerwartung, und die Präferenz des Investors zeigt, welches die nutzenmaximale Kombination von Risiko und Renditeerwartung ist.

- Die zweite, davon gelöste und gleichsam untergeordnete Aufgabe verlangt dann in jedem Augenblick, das "Sigma optimal zu verwalten." Die einzelnen Wertpapiere werden dazu so gewichtet und kombiniert, dass ein effizientes Portfolio mit eben dieser Volatilität Sigma zustande kommt. Änderungen und Anpassungen, auch beispielsweise ein "Balancing", können (wenn Transaktionskosten wegfallen) augenblicklich vollzogen werden. Für diese Aufgabe der optimalen Verwaltung von Sigma kommt dann in jedem Augenblick ein effizientes Portfolio in Frage.

5 Zum Lernen im Zusammenhang mit der dynamischen Portfolio-Selektion vergleiche Brennan (1998). 
Nach dieser Vorklärung konzentrieren wir uns auf die erste Aufgabe, die Optimierung des Exposures über die Zeit. Der Blick muß auf die "Zielfunktion" gerichtet werden Aus Sicht der ökonomischen Theorie sollte das Ziel die Maximierung des Erwartungsnutzens sein, der mit dem unsicheren Endvermögen verbunden ist, welches mit Ende des Anlage- und Planungszeitraums für Konsum und andere Zwecke (etwa Vererbung) zur Verfügung steht. Wir behandeln die Aufgabe der Strategischen Asset Allocation demnach als die Aufgabe, das Exposure - das möglicherweise mit der Zeit und zwischenzeitlichen Zuständen (augenblickliches Vermögen) variiert - zu bestimmen, so dass der Erwartungsnutzen des unsicheren Endvermögens maximal wird. ${ }^{6}$

Bei diesem Denkrahmen öffnet sich ein breiter Raum für die Modellierung der Risikonutzenfunktion. Es genügt dann nicht mehr, pauschal über die "Höhe der Risikoaversion" oder die Stärke der Risikotoleranz" zu sprechen. Gerade bei einem langjährigen Anlagehorizont verändert sich das (nominale) Finanzvermögen deutlich über die Zeit. Es genügt dann nicht mehr, die Höhe der Risikoaversion der Person als eine Zahl zu bestimmen, denn es kommt gleichermaßen auf die Abhängigkeit ihrer Risikoaversion vom Vermögen an. Das ist ein wesentlicher Unterschied zur Portfolio-Selektion für den Horizont eines Jahres. Die Literatur zur Anwendung der Expected Utility Theory auf die Portfolioselektion hat verschiedene Verläufe für die Risikoaversion vorgeschlagen, und jeder Verlauf oder jede Risikonutzenfunktion zieht eine eigene dynamische Anlagepolitik nach sich. Vereinfacht können die Ergebnisse hierzu so formuliert werden: Wenn die absolute Risikoaversion einer Person konstant (mit dem Vermögen) ist, möchte diese Person im wesentlichen denselben Geldbetrag in Aktien halten (ungeachtet davon, wie sich die Höhe ihres Vermögens im Verlauf der Zeit entwickelt). ${ }^{7}$ Diese Person wird, wenn sich mit den Jahren das Vermögen entwickelt, jedenfalls einen immer geringeren Anteil risikobehaftet anlegen. Wenn hingegen die relative Risikoaver-

6 Von einem "Nutzen", der von dem zwischenzeitlichen Erreichten ausgeht, wird abgesehen. Alternativ dazu könnten zwischenzeitliche Konsumentnahmen berücksichtigt werden, die einen Nutzenbeitrag liefern. Diese Modellierung geht auf Merton $(1969,1971)$ zurück. Es wird dann der Lebensnutzen modelliert, der sich aus dem Konsum ableitet, der in den verschiedenen Jahren über den Lebenszyklus hinweg möglich ist. Der Konsum wird mit Hilfe von exogenen Arbeitseinkünften einer endogenen Politik der Investition und Desinvestition finanziert. Hierbei sind die zentralen Einflußgrößen des nutzenmaximalen Konsum- und Investitionsplans die Risikoaversion des Individuums und die Zeitpräferenz (Geduld) hinsichtlich des Konsums. Diesen Ansatz aufgreifend, zeigen Loewenstein/Prelec (1993), dass Menschen im Verlauf der Zeit periodisch ihr Nutzenniveau beurteilen und es ihre Zielsetzung ist, einen steigenden Nutzen zu haben.

7 Allerdings gilt das nicht ganz exakt, weil sich im Verlauf der Zeit der Typus der Wahrscheinlichkeitsverteilung des Endvermögens ändert, denn typischerweise wird die Rechtsschiefe immer ausgeprägter. 
sion eines Investors konstant ist, möchte er stets denselben Anteil seines Vermögens in Aktien halten.

Die Erkenntnis, dass jede Risikonutzenfunktion eine eigene Dynamik hinsichtlich der Geldanlage über die Zeit impliziert, ist von großer Bedeutung für die Praxis. In der Vermögensverwaltung ermitteln Berater durch Befragungen lediglich die "Höhe" der Risikoaversion eines Kunden (an der Stelle des augenblicklichen Vermögens) und versuchen erst gar nicht herauszufinden, wie hoch die Risikoaversion des Kunden wäre, wenn das Vermögen vielleicht fünfmal so hoch wäre - obwohl diese Situation vermutlich in zwanzig Jahren eingetreten ist. Gerade die Information, wie sich die Risikoaversion mit dem Vermögen ändert, ist aber für eine korrekte Bestimmung der Dynamik des optimalen Risikoexposures wichtig.

Im Folgenden werden wir das Modell von Samuelson (1989) in den Mittelpunkt rücken. Es postuliert konstante relative Risikoaversion. Andere Autoren haben eine empirische Bestätigung für diese Annahme geliefert oder die Höhe dieser konstanten relativen Risikoaversion geschätzt. Das Samuelson-Modell gestattet eine analytische Herleitung der optimalen Anlagepolitik. Ein über die Zeit hinweg konstantes Exposure erweist sich als richtig: Der (prozentuale) Anteil des Vermögens, der risikobehaftet angelegt werden soll, gleichsam die Aktienquote, ist über die Zeit hinweg konstant. Insbesondere wird die Aktienquote nicht reduziert, wenn der Investor immer näher an das Ende des Anlagezeitraums kommt und nun das Ergebnis (für Konsumzwecke) entnehmen möchte. Die Aktienquote wird auch nicht nach einer Hausse oder nach einer Baisse korrigiert. Noch dazu ist die Höhe der optimalen Aktienquote im Samuelson-Modell unabhängig von der Gesamtdauer der Geldanlage. Dieses formal bewiesene Ergebnis widerspricht der Intuition, wo viele Berater immer wieder dies betonen: Ein Engagement in Aktien setzt einen entsprechend langen Horizont voraus, und wenn im Verlauf der Anlage der Fälligkeitstermin näher rückt, sollte die Aktienquote reduziert werden. Indessen liegt kein Widerspruch vor. Die Auflösung des Puzzels liegt in der Erfordernis, das im Samuelson-Modell unterstellte Vermögen aus der finanziellen Situation des Investors abzuleiten. Bereits fest verplante Teile des Anlageergebnisses eine Lebenshaltungsverpflichtung (subsistence) des Investors sich selbst und seiner Familie gegenüber - verlangen, dass ihr Present Value schon zum Zeitpunkt der Geldanlage "auf die Seite gelegt" und zum Zinssatz angelegt wird und so von der weiteren Betrachtung ausgeklammert bleibt. Gleiches gilt für sonstiges Vermögen, über das dem Investor am Ende der Anlagedauer neben dem Ergebnis der Finanzanlage zur Verfügung steht.

Damit wird ist eigentlich nicht die Länge der Anlagedauer an sich wichtig, wohl aber, ob der Investor zum Zeitpunkt der geplanten "Auflösung" des Ergebnisses der Finanzanlage noch auf sonstiges Vermögen blicken kann oder ob dann vielleicht sogar eine Verpflichtung besteht. Ein Investor kann sein Geld deutlich riskanter anlegen, wenn zum Ende des Anlagezeitraums sonst "noch etwas da" ist, und ein anderer Investor mit derselben Risikoaversion muß sein Geld deutlich 
vorsichtiger anlegen, wenn zum Ende des Anlagezeitraums "Verpflichtungen" bestehen. Wichtig: es kommt nicht darauf an, wie sich hinter dem sonstigen Vermögen stehende Positionen (Humankapital, Ansprüche aus Pensionssicherung und dergleichen) vorher verhalten - es kommt nur auf ihren Wert zum Zeitpunkt an, zu dem die Geldanlage in der Planung als beendet betrachtet wird. Ähnliches gilt für Verpflichtungen. Nur ihr Wert zum Ende des Anlagehorizonts ist von Bedeutung für die Aktienquote. Fast sind diese Einflüsse bedeutender als der Einfluss der Risikoaversion. Der Berater sollte daher, wenn das Geld einer Person anzulegen ist nicht dies fragen: "In wieviel Jahren wollen Sie das Geld wiederhaben und wie hoch ist ihre Risikotoleranz." Statt dessen sollte der Berater diese Frage stellen: "Wenn Sie Ihr Geld mit Rendite wiederhaben wollen — wann immer das genau sein wird - werden Sie dann außerdem noch anderes Vermögen haben oder bestehen dann vielleicht sogar Verpflichtungen?"

\section{Erwartungsnutzen}

Das Grundmodell: Eine Person soll zum heutigen Zeitpunkt $t=0$ einen gegebenen Geldbetrag anlegen. Außerdem soll sie den Zeitpunkt $T$ kennen, zu dem das Anlageergebnis zur Verfügung stehen soll. Jede (statische oder dynamische) Politik der Anlage bewirkt ein unsicheres Anlageergebnis zu diesem Zeitpunkt, und aufgrund der als bekannt vorausgesetzten stochastischen Eigenschaften der einbezogenen Anlageinstrumente kann für jede Anlagepolitik die Wahrscheinlichkeitsverteilung des Ergebnisses ermittelt werden. Die Wahl der Anlagepolitik entspricht so vor allem einer Auswahl aus mehreren Verteilungen des Anlageergebnisses.

Personen, die angesichts unsicherer Ergebnisse Entscheidungen treffen, folgen letztlich einer persönlichen Präferenz. Wenn diese Präferenz gewisse Eigenschaften erfüllt, die in Kreisen der Wissenschaft immer als Axiome rationaler Entscheidungen apostrophiert wurden, ${ }^{8}$ läßt sich die Präferenz auf eine besondere funktionale Weise darstellen: Es gibt eine sogenannte Risikonutzenfunktion, $u$, und die Person wählt aufgrund ihrer Präferenz jenes Ergebnis (und damit jene Anlagepolitik), das den Erwartungsnutzen der Ergebnisse maximiert. Die Person entscheidet so, als $o b$ sie den Erwartungsnutzen maximieren würde. ${ }^{9}$

Wichtig ist: Das Argument der Risikonutzenfunktion ist das totale Endvermögen der Person und nicht nur die durch die Entscheidung herbeigeführte Änderung des

Zur Axiomatik der Expected Utility Theory siehe etwa Dréze (1974).

9 Das Entscheidungskriterium des Erwartungsnutzens ist bereits 1738 von Daniel Bernoulli postuliert worden - er wählte als Risikonutzenfunktion in seinen Beispielen den Logarithmus. 
anfänglichen Vermögens. ${ }^{10}$ Das totale Endvermögen (das Argument der Risikonutzenfunktion) kann als Summe des Anlageergebnisses und eines sonstigen Vermögens dargestellt werden - dabei wird es sich um Vermögenspositionen handeln, die üblicherweise nicht in die Anlageentscheidung einbezogen sind: $\mathrm{Hu}-$ mankapital, Immobilien, und so fort. Beträgt das sonstige Vermögen der Person zum Zeitpunkt $T$ dann $w_{T}$, und stehen zwei Anlagepolitiken zur Wahl, die aus dem anfänglichen Finanzvermögen die unsicheren Ergebnisse $\widetilde{y}$ beziehungsweise $\widetilde{z}$ entstehen lassen, so wird die Person genau dann $\widetilde{z}$ vorziehen, wenn

$$
E\left[u\left(w_{T}+\widetilde{z}\right)\right]>E\left[u\left(w_{T}+\widetilde{y}\right)\right]
$$

gilt. Eine leicht ableitbare Größe ist das Sicherheitsäquivalent eines Finanzergebnisses oder einer Vermögensänderung - kurz einer Lotterie - wie $\tilde{y}$ oder $\widetilde{z}$. Das Sicherheitsäquivalent einer Lotterie $\widetilde{z}$ ist jener sichere Geldbetrag $c$, bei der die Person indifferent zwischen $\widetilde{z}$ und der sicheren Vermögensänderung $c$, also

$$
E\left[u\left(w_{T}+\widetilde{z}\right)\right] \equiv E\left[u\left(w_{T}+c\right)\right] .
$$

Hier stimmt die rechte Seite mit $u\left(w_{T}+c\right)$ überein, wenn neben der sicheren GröBe $c$ auch $w_{T}$ als sicher angesehen wird. Ist die Risikonutzenfunktion streng monoton, folgt demnach für das Sicherheitsäquivalent

$$
c=u^{-1}\left\{E\left[u\left(w_{T}+\widetilde{z}\right)\right]\right\}-w_{T}
$$

Für wirtschaftlich relevante Entscheidungen darf Risikoaversion (RA) unterstellt werden: Wenn sie die Wahl hat, zieht die Person einer jeden unsicheren Zahlung stets eine sichere Zahlung in Höhe des Erwartungswerts der Zahlung vor. Anders ausgedrückt: Risikoaversion bedeutet: das Sicherheitsäquivalent $c$ einer unsicheren Vermögensänderung $\widetilde{z}$ ist stets geringer als ihr Erwartungswert, $c<E[\widetilde{z}]$.

Die Risikoaversion zeigt sich in der Konkavität der Risikonutzenfunktion, und ein Maß der Risikoaversion leitet sich aus der Konkavität, also der zweiten Ableitung der Risikonutzenfunktion ab. Arrow sowie Pratt (1964) haben als Maß die absolute Risikoaversion (ARA) eingeführt,

$$
A R A \equiv-\frac{u^{\prime \prime}(w)}{u^{\prime}(w)} .
$$

Der Kehrwert der ARA wird als Risikotoleranz bezeichnet. Arrow und Pratt haben gezeigt, was diese Definition leistet: Für alle Risikonutzenfunktionen (unter gewissen Differenzierbarkeitsbedingungen) ist das Sicherheitsäquivalent einer unsi-

10 Das ist ein wesentlicher Unterschied zwischen der Expected Utility Theory und dem Kriterium von Kahneman/Tversky (1979), in dem die Änderung des Vermögens den wahrgenommenen Nutzen beeinflußt. 
cheren Lotterie gleich ist dem Erwartungswert minus das $A R A$ / 2 -fache der Varianz,

$$
c \approx E[\widetilde{z}]-\frac{A R A}{2} \cdot \operatorname{Var}[\widetilde{z}]
$$

Im hybriden Modell gilt diese Beziehung sogar exakt und nicht nur approximativ. ${ }^{11}$ Entsprechend wird eine Person, die Geld auf ein Jahr anlegt, und hierbei eine sichere Anlage mit der Rendite $r_{f}$ und eine unsichere Anlage mit der Renditeerwartung $\mu$ und der Renditestreuung $\sigma$ wählen kann, den Geldbetrag

$$
x=\frac{\mu-r_{f}}{A R A \cdot \sigma^{2}}
$$

risikobehaftet anlegen. Zu den Einheiten: Das Produkt aus ARA und dem Vermögen ist eine Zahl, weshalb die ARA in der Einheit 1/Geldeinheit gemessen wird. Ist die Geldeinheit 1 Million Euro, könnte die ARA einer Person beispielsweise 2 [1/(Million Euro)] betragen. Gilt für den Erwartungswert der einfachen (diskreten) Aktienrendite $\mu=9,4 \%$, weiter $\sigma=20 \%$ und für den Zinssatz $r_{f}=4,6 \%$, dann legt diese Person $x=(0,094-0,046) / 2 \cdot 0,04=0,6$ [Million Euro] in Aktien an. Würde man nun als Geldeinheit 1 Tausend Euro wählen, würde die ARA derselben Person 0,002 [1/(Tausend Euro)] betragen. Für die unveränderten Daten von $\mu, r_{f}$ und $\sigma$ legt die Person nun $x=0,048 / 0,002 \cdot 0,04=600$ [Tausend Euro] in Aktien an.

Sofern der Anlagehorizont etwa ein Jahr (und nicht wesentlich länger) beträgt, wird die optimale Aktienquote zwar durch die ARA bestimmt, nicht aber durch den genauen Typ von Risikonutzenfunktion. Praktisch alle Risikonutzenfunktionen, sofern sie an der Stelle des heutigen Vermögens dieselbe ARA aufweisen, führen auf praktisch dieselbe Aktienquote - wie auch immer ihr genauer Verlauf ist. Bei einer kurzfristigen Geldanlage (beispielsweise auf ein Jahr) ist es daher erstaunlicherweise unbedeutend für die Aktienquote, wie der genaue Typ der Risikonutzenfunktion ist; die Aktienquote ist nur durch die ARA bestimmt. Eine weitere Frage lautet, welcher Nutzennachteil entsteht, wenn von der optimalen Aktienquote abgewichen wird. Wie Brennan und wie Spremann (2003) ausführen, ist im Fall der Einjahresanlage kein nennenswerter Unterschied im Nutzen (ausgedrückt durch das Sicherheitsäquivalent) festzustellen: Das Risikoexposure spielt bei der Anlage auf ein Jahr in der Nutzenbilanz nicht die große Rolle, die ihr von Beratern immer zugewiesen wird: Alle Aktienquoten bewirken praktisch denselben Nutzen.

11 Das hybride Modell besteht in der Verbindung der Annahme normalverteilter Lotterien und konstanter ARA, vergleiche Bamberg/Spremann (1981). 
Diese Ergebnisse lassen sich jedoch nicht auf eine mehrjährige Anlage übertragen. Zunächst fällt auf, dass die Risikoaversion durch die Ableitungen der Risikonutzenfunktion an der Stelle $w$ beschrieben sind und sich daher auf ein Argument beziehen. So stellt sich die Frage, wie sich die RA mit zunehmendem $w$ verhält. Denn im Verlauf einer mehrjährigen Anlage verändert sich natürlich das Vermögen stärker. Meistens wächst es (nominal) an. Allgemein wird aufgrund zahlreicher Experimente und Beobachtungen von Menschen die Vorstellung geteilt, dass $A R A(w)$ mit $w$ eher abnimmt als zunimmt. Wer 1 Million Euro auf 20 Jahre anlegt - und das unsichere, nach 20 Jahren erzielte Ergebnis mit einer Risikonutzenfunktion bewertet - muß beachten, dass in 20 Jahren vielleicht 5 Millionen Euro zu erwarten sind und dass es für die Strategische Asset Allocation deshalb darauf ankommt, welche ARA die Person an der Stelle $w=5$ [Million Euro] hat. Zur Beschreibung dieser Veränderlichkeit der ARA mit dem Vermögen werden in der Literatur Klassen von Funktionen vorgeschlagen.

Bei der einen Klasse wird die ARA als konstant mit dem Vermögen angenommen, $A R A(w) \equiv \alpha$, und folglich werden diese Risikonutzenfunktionen als CARA (constant absolute risk aversion bezeichnet). Die Klasse CARA enthält für $\alpha>0$ die negative Exponentialfunktion, $u(w)=-\exp (-\alpha \cdot w)$, sowie für $\alpha=0$ die lineare Risikonutzenfunktion $u(w)=w$, welche Risikoneutralität beschreibt. Eine Person, die CARA aufweist, trifft Anlageentscheidungen ohne Bezug auf ihr sonstiges Vermögen. Das Sicherheitsäquivalent ist vom Vermögen unabhängig. ${ }^{2}$ Zwei Menschen mit übereinstimmender konstanter ARA (und demselben Anlagehorizont) halten daher denselben Betrag in Aktien, auch wenn sie ganz unterschiedliche Gesamtvermögen haben. Das erscheint wenig einsichtig, doch ließe sich dieses formal bewiesene Ergebnis für die praktische Interpretation noch heilen, indem im Kollektiv der Investoren ein gegenläufiger Zusammenhang zwischen dem Gesamtvermögen des Investors und der Höhe seiner ARA hergestellt wird. Wer an CARA festhält, wird demnach unterstellen, dass ein Armer eines höheres $\alpha$ hat als ein Reicher. Das ist ein möglicher Weg, um die Klasse CARA doch noch als realitätskonform akzeptieren zu können. Allerdings war der Reiche vielleicht früher einmal arm und ist nur im Verlauf seines Lebens durch eine rentable Geldanlage reich geworden. Seine ARA müßte daher doch irgendwie vom Vermögen abhängen und dürfte folglich nicht konstant sein. Die Nutzenfunktion CARA eignet sich daher wenig für die Beschreibung der Ziele langfristiger Geldanlage (bei der im Verlauf der Zeit anfänglich Arme reich werden) - auch wenn sie in einem kurzfristigen Kontext alle anderen Risikonutzenfunktionen hinreichend genau approximiert, wie Samuelson zeigte.

Eine weitere Klasse von Risikonutzenfunktionen nimmt an, die absolute Risikoaversion $A R A(w)$ verlaufe umgekehrt proportional zu $w, A R A(w)=(1-\gamma) / w$. Anders ausgedrückt wird postuliert, dass die relative Risikoaversion,

12 Schneeweiß (1967). 


$$
R R A(w) \equiv w \cdot A R A(w),
$$

konstant ist: $R R A(w) \equiv 1-\gamma$ (es ist üblich, für 1-RRA den Parameter $\gamma$ zu verwenden). Die RRA ist eine dimensionslose Zahl, invariant gegenüber Änderung der Einheit, in der Geldbeträge ausgedrückt werden. Die Klasse von Risikonutzenfunktionen mit konstanter relativer Risikoaversion wird mit CRRA bezeichnet. Für $\gamma=0$, also für $R R A(w) \equiv 1$, ist die entsprechende Risikonutzenfunktion der Logarithmus. Für $\gamma \neq 0$ enthält die Klasse CRRA die Exponentialfunktionen

$$
u(w)=\frac{1}{\gamma} w^{\gamma}
$$

Beispiele: A) Für $\gamma=1 / 2$, also $R R A(w) \equiv 1 / 2$ (ungeachtet der Frage, ob die Geldeinheit Euro, Tausend Euro oder Millionen Euro ist), was eine recht risikotolerante Person ist, gilt $u(w)=2 \cdot \sqrt{w}$. B) Für für $\gamma=-1$, also $R R A(w) \equiv 2$ (das ist eine Person mit einer "typischen" Risikoaversion), gilt $u(w)=-1 / w$. C) Für $\gamma=-2$, also $R R A(w) \equiv 3$ (das ist eine Person mit einer leicht höheren aber immer noch öfters anzutreffenden Risikoaversion), gilt $u(w)=-1 /\left(2 \cdot w^{2}\right)$.

Blume/Friend (1975), Friend/Blume (1975) und Friend (1977) haben in groß angelegten Erhebungen gefunden, dass (amerikanische) Haushalte mit höherem Vermögen tendenziell auch höhere Beträge risikobehaftet investiert halten. Die Daten legen nahe, dass für jeden Haushalt die relativen Anteile der Aktienbestände am Gesamtvermögen annähernd konstant bleiben. Somit sprechen diese Studien dafür, Modellen die Risikonutzenfunktionen vom Typ CRRA zugrunde zu legen. Friend (1977) sieht $R R A \geq 2$ als Regelfall an $^{13}$ und bezeichnet Personen mit $R R A \leq 1$ als Ausnahme. Der Logarithmus als Risikonutzenfunktion beschreibt daher den Grenzfall eines sehr risikotoleranten Investors. ${ }^{14}$ Andere Studien gelangen zu einer ähnlichen Favorisierung der Klasse CRRA. Pindyck (1988) schätzt $3 \leq R R A \leq 4$. In Arbeiten, die das Equity-Premium-Puzzle erklären wollen, wird eine sehr hohe RRA angenommen: Grossman/Shiller (1981) gehen von $6 \leq R R A$ aus.

\section{Das Samuelson-Modell}

Für eine Risikonutzenfunktion vom Typ CCRA kann das Problem der Optimierung des Exposures so formuliert werden: Gegeben ist ein Startbetrag $w_{0}$, der zur Zeit 0 angelegt werden soll. Gegeben ist auch der Anlagehorizont $T$, und das

13 In Verbindung mit realitätsnahen Finanzmarktdaten führt dies auf einen Aktienanteil um $30 \%$ bis $45 \%$.

14 Interessanterweise sieht Friend (1977) institutionelle Investoren als noch risikoaverser an im Vergleich zu Privatinvestoren. 
dann erreichte Vermögen stiftet für den Investor Nutzen. Der Investor habe eine $R R A \equiv 1-\gamma$, das heißt, die Risikonutzenfunktion $u(w)=(1 / \gamma) \cdot w^{\gamma}$. Alle Anlagepolitiken werden anhand des Erwartungsnutzens des totalen Endvermögens evaluiert.

Als Anlagevehikel bietet sich erstens eine risikofreie Anlage, die durch den stetigen Zinssatz $r_{f}$ beschrieben ist. Dieser soll im Zeitintervall $0 \leq t \leq T$ als konstant angenommen werden. Wird also zu $t=0$ der Betrag 100 risikofrei angelegt und bis $T$ gehalten, ist er auf $100 \cdot \exp \left(T \cdot r_{f}\right)$ gewachsen. Beispielsweise beträgt die durchschnittliche Rendite, die 1926-2003 mit Staatsanleihen Schweiz erzielt wurde, 4,6\% . Bei einer langfristigen Anlage kann dies durchaus als die Rendite einer sicheren Anlage dienen. Wer den Startbetrag über $T=20$ Jahre so anlegt, kann mit dem sicheren Anlageergebnis $100 \cdot 1,046^{20}=246$ rechnen. Da $r_{f}$ in stetiger Notation angegeben ist, folgt $r_{f}=\ln (1,046)=4,5 \%$. In der Tat ergibt auch $100 \cdot \exp \left(T \cdot r_{f}\right)=100 \cdot \exp (20 \cdot 0,045)=246$.

Zweitens steht eine risikobehaftete Anlage zur Verfügung, deren Wertentwicklung durch die stochastische Differentialgleichung

$$
\frac{d s}{s}=\mu \cdot d t+\sigma \cdot d z
$$

beschrieben ist. Der Drift der relativen Wertänderung, $\mu$, und die Volatilität, $\sigma$, sollen gegebene Konstanten sein. Hinweis: Eine andere, ebenso übliche Beschreibung des stochastischen Prozesses der Wertentwicklung stellt den Prozeß der stetigen Gesamtrendite in den Mittelpunkt:

$$
d r=\mu^{*} \cdot d t+\sigma \cdot d t
$$

Die Wertentwicklung wird demnach durch eine geometrische Brownsche Bewegung beschrieben - ein Standardmodell im Finance. Der Zusammenhang zwischen dem oben eingeführte Drift der relativen Wertänderung, $\mu$, und dem Erwartungswert der stetigen Rendite, $\mu *$, wird durch das Lemma von Itô vermittelt und lautet:

$$
\mu^{*}=\mu-\frac{\sigma^{2}}{2}
$$

Die Volatilitäten beider Prozesse stimmen überein. ${ }^{15}$ Eine mit dem Startbetrag $s_{0}$ zu $t=0$ beginnende Anlage in diesen Proze $\beta$ hat also zum Endzeitpunkt $T$ das

15 Allerdings ist die Volatilität der diskreten (einfachen) Renditen höher. 
unsichere Ergebnis $\widetilde{s}_{T}$, und dessen Verteilungsparameter Erwartungswert $E$, Median $M$, Modus $L$ sind gegeben durch:

$$
\begin{gathered}
E\left[\widetilde{s}_{T}\right]=s_{0} \cdot \exp (\mu \cdot T)=s_{0} \cdot \exp \left(\left(\mu *+\frac{1}{2} \cdot \sigma^{2}\right) \cdot T\right) \\
M\left[\widetilde{s}_{T}\right]=s_{0} \cdot \exp \left(\left(\mu-\frac{1}{2} \cdot \sigma^{2}\right) \cdot T\right)=s_{0} \cdot \exp (\mu * \cdot T) \\
L\left[\widetilde{s}_{T}\right]=s_{0} \cdot \exp \left(\left(\mu-\frac{3}{2} \cdot \sigma^{2}\right) \cdot T\right)=s_{0} \cdot \exp \left(\left(\mu *-\sigma^{2}\right) \cdot T\right)
\end{gathered}
$$

Wenn eine Anlage in diese Prozesse die Volatilität $\sigma$ aufweist, dann hängt gemäß der obersten dieser drei Gleichungen der Erwartungswert der diskreten oder einfachen Jahresrendite, $\mu_{\text {ein }}$, mit dem Drift der relativen Wertänderung so zusammen: $1+\mu_{\text {ein }}=\exp (\mu)$, also $\ln \left(1+\mu_{\text {ein }}\right)=\mu$. Beispiel: Die durchschnittliche diskrete Jahresrendite auf Aktien Schweiz (1926-2003) beträgt 9,4\%, und diesen Mittelwert setzen wir mit $\mu_{\text {ein }}$ gleich. Dann hat der Prozeß der relativen Wertänderung den Drift $\mu=9,0 \%$. Die Volatilität liegt ziemlich genau bei $\sigma=18 \%$. Der Erwartungswert der stetigen Rendite beträgt $\mu^{*}=9 \%-(0,18 \cdot 0,18 / 2)=7,4 \%$. Das ist zugleich, in stetiger Notation, die Medianrendite. Das heißt: Eine Anlage $s_{0}=100$ führt nach einem Jahr $(T=1)$ auf ein stochastisches Ergebnis $\widetilde{s}_{1}$, und die Wahrscheinlichkeit ist jeweils $50 \%$ dass es über beziehungsweise unter dem Median $M_{1}=100 \cdot \exp (0,074)=107,68$ liegt. Die Modalrendite in stetiger Notation beträgt hingegen nur $\mu^{*}=9,0 \%-(0,18 \cdot 0,18 \cdot 3 / 2)=4,14 \%$. Das heißt: Eine Anlage $s_{0}=100$ führt nach einem Jahr $(T=1)$ auf ein stochastisches Ergebnis $\widetilde{s}_{1}$, und der häufigste Wert (Maximum der Wahrscheinlichkeitsdichte) ist gerade einmal $L_{1}=100 \cdot \exp (0,0414)=104,23$.

Der Investor wählt nun sein Exposure $x_{t}$ für alle Zeitpunkte $0 \leq t \leq T$. Dieses Exposure $x_{t}$ ist der Anteil des zu $t$ erreichten Vermögens $w_{t}$, der risikobehaftet investiert ist. Der restliche Anteil $1-x_{t}$ wird sicher angelegt. Die relative Änderung dieses Vermögens hat demnach den Drift $r_{f}+x_{t} \cdot\left(\mu-r_{f}\right)$ und die Volatilität $x_{t} \cdot \sigma$. Der Prozeß der Vermögensentwicklung folgt somit der stochastischen Differentialgleichung

$$
\frac{d w}{w}=\left(r_{f}+x_{t} \cdot\left(\mu-r_{f}\right)\right) \cdot d t+\left(x_{t} \cdot \sigma\right) \cdot d z
$$

Samuelson (1989) hat gezeigt, wie die optimale Politik $x_{t}, 0 \leq t \leq T$, aussieht: 


$$
x_{t}=\frac{\mu-r_{f}}{(1-\gamma) \cdot \sigma^{2}}
$$

Die optimale Aktienquote ist erstens von $t$ unabhängig — sie ist über die Zeit hinweg konstant. Insbesondere ändert sich die optimale Aktienquote nicht, wenn $t$ näher an $T$ herankommt. Zweitens hängt die optimale Aktienquote auch nicht von $T$ ab, der Gesamtdauer der Geldanlage. Sie ist bestimmt nur durch a) die Überrendite $\mu-r_{f}$, b) die Höhe der konstanten RRA, $1-\gamma$, c) sowie die Volatilität. Damit diese Politik durchgehalten wird, muß der Investor (sofern nicht gerade $x_{t} \equiv 1$ ist) laufend "rebalancieren,", da sich in jedem Augenblick die Aktien anders entwickeln als die sichere Anlage. Die tatsächliche Aktienquote erhöht sich natürlich, wenn die Aktien gut laufen, und zum Balancing muß der Investor dann einige Aktien verkaufen und den Erlös sicher anlegen. Wenn umgekehrt die Aktien schlecht abschneiden, muß der Investor Geldmarktinstrumente und Bonds verkaufen und Aktien hinzukaufen. Das Balancing erfordert klar eine antizyklische Adjustierung.

Zurück zur Formel für das optimale Exposure: Mit den zuvor genannten empirischen Schätzungen $\mu=0,090, r_{f}=0,045, \sigma=0,18$ hat ein Investor mit der relativen Risikoaversion 2 oder $\gamma=-1$ stets $69,4 \%$ seines Vermögens risikobehaftet angelegt. Gemäß der Querschnittsuntersuchung von Friend (1975) wäre ein Investor mit $R R A=1$, also $\gamma=0$, ein (selten anzutreffender) Grenzfall hin zur Seite hoher Risikotoleranz. Dieser Investor würde rechnerisch 138,9\% seines Vermögens in Aktien halten. Das heißt, ein vollständiges Aktienexposure wäre ihm nicht rentabel genug und er wäre zu mehr Risiko bereit, als mit $\sigma=18 \%$ verbunden ist. Solche Personen gibt es vereinzelt in der Wirklichkeit: sie legen in Private Equity an, betätigen sich unternehmerisch, oder nehmen einen Lombardkredit. Gemäß der Studie von Friend (1975) sind sie aber untypisch und man wird häufiger Personen mit $R R A=2$ oder höher anzutreffen. Ein Investor mit der relativen Risikoaversion $4(\gamma=-3)$ hat optimalerweise $34,7 \%$ seines Vermögens risikobehaftet angelegt und ein Investor mit $R R A=6$ wählt $23,1 \%$. Wenn Grossman/Shiller (1981) von $6 \leq R R A$ ausgehen, dann heißt das: Die meisten Menschen wünschen sich eine Aktienquote, die $23,1 \%$ oder geringer ist. Ein Investor mit $R R A=8$ wählt $17,4 \%$ und ein Investor mit $R R A=10$ wählt $13,9 \%$ als Aktienquote.

Ein "rationaler" Investor wird so wählen, als ob er den Erwartungsnutzen zu maximieren versucht. Indessen könnte es auch Investoren geben, die nicht die Wahrscheinlichkeitsverteilung des späteren Endvermögens betrachten und diese durch den Erwartungsnutzen bewerten, sondern die gern ihre Strategie danach ausrichten, was sie denn später "konkret" erhalten. Das Anlageergebnis $\widetilde{w}_{T}$ ist (bei einer konstanten Aktienquote) lognormalverteilt und weist damit eine ausgeprägte 
Rechtsschiefe aus, besonders wenn der Anlagezeitraum lang und die Aktienquote hoch ist. Indessen werden manche Investoren nach dem wahrscheinlichsten Wert fragen, den das Endergebnis annehmen wird, und das ist der Modus von $\widetilde{w}_{T}$. Einzelne Investoren werden also eine Anlagestrategie wählen, die den Modus als den "wahrscheinlichsten" Wert des Endergebnisses maximiert. Sie weichen von den Axiomen der Expected Utility Theory ab; bei einer solchen Zielsetzung kommt es auf eine Risikonutzenfunktion überhaupt nicht mehr an und auch die Risikoaversion des Investors muß nicht bestimmt werden. Zur Illustration wollen wir die entsprechenden Rechnungen mit den konkreten Finanzmarktdaten vorführen. Dazu unterstellen wir, dass diese Investoren die Politik übernehmen, einen konstanten Anteil des Vermögens risikobehaftet anzulegen, die sich im Fall der Maximierung des Erwartungsnutzens durch die Rechnungen von Samuelson als optimal herausgestellt haben. Für die Anlagepolitik $x_{t} \equiv x$ beträgt, ausgehend vom Anfangsver-

mögen $w_{0}$, der Modus $L_{T}=w_{0} \cdot \exp \left(\left(r_{f}+x \cdot\left(\mu-r_{f}\right)-x^{2} \cdot \sigma^{2} \cdot 3 / 2\right) \cdot T\right)$. Diese Zielfunktion wird maximiert für

$$
x_{\text {Max-Modus }}=\frac{\mu-r_{f}}{3 \cdot \sigma^{2}}
$$

Für die genannten Finanzmarktdaten ist es also eine Aktienquote 46,3\% jene, die das wahrscheinlichste Endergebnis möglichst groß werden läßt. Das gleiche Exposure wählt übrigens ein nach den Axiomen des Erwartungsnutzen handelnder Investor, wenn er eine konstante relative Risikoaversion $R R A=3$ besitzt. Ist eine Person nicht in der Lage, die Höhe ihrer Risikoaversion zu artikulieren, wäre also die Empfehlung mit $R R A=3 \mathrm{zu}$ rechnen, denn dafür ergibt sich eine Anlagestrategie, die das wahrscheinlichste Endergebnis möglichst groß werden läßt. Wenn ein Investor den Berater fragt "wie risikotolerant empfehlen Sie mir denn zu sein?" so könnte dieser vor dem Hintergrund dieser Betrachtung antworten: "Entscheiden Sie doch so, als wenn Sie eine konstante relative Risikoaversion von 3 hätten.”

\section{Gesamtbilanz}

In der Expected Utility Theory ist das Argument der Risikonutzenfunktion das totale Endvermögen zum Zeitpunkt $T$. Die Darstellung des Samuelson-Modells war so formuliert, als ob das totale Endvermögen und das Ergebnis der Finanzanlage $\widetilde{w}_{T}$ übereinstimmen. In vielen und praxisnahen Fällen wird das totale Vermögen der Person zum Zeitpunkt $T$ neben dem Finanzvermögen $\widetilde{w}_{T}-$ dessen Verteilung durch die Anlagepolitik bestimmt wird - noch weiteres, nicht disponibles Vermögen umfassen. Dieses sonstige Vermögen (zum Zeitpunkt $T$ ) soll mit $v_{T}$ bezeichnet werden. Das totale Endvermögen beträgt also $\widetilde{w}_{T}+v_{T}$. 
Zum sonstigen Vermögen gehören alle Positionen, die neben dem Finanzvermögen $\widetilde{w}_{T}$ zum Zeitpunkt $T$ der Person zur Verfügung stehen, und wie der Konsum des Anlageergebnisses $\widetilde{w}_{T}$ Nutzen stiften. Zum sonstigen Vermögen gehört beispielsweise eine Immobilie, die sich zum Zeitpunkt $T$ im Eigentum der Person befindet. Falls die Person nach dem Zeitpunkt $T$ noch (weitere, dann folgende) Arbeitseinkünfte beziehen wird, oder Ansprüche auf Pensionsleistungen hat, dann zählen auch die auf $T$ bezogenen Barwerte dieser nach $T$ noch folgenden Einnahmen zum sonstigen Vermögen $v_{T}$. Wir werden weiter unten auch Verpflichtungen behandeln, die bewirken, dass das sonstige Vermögen negativ oder jedenfalls reduziert ist. Die Berücksichtigung all dieser Positionen geschieht in Form einer Gesamtbilanz.

Wir wollen untersuchen, wie diese Gesamtbilanz formal aussieht. Dabei wird sich zeigen, dass es auf den Barwert ankommt, den das sonstige Vermögen $v_{T}$ zu den Zeitpunkten $t<T$ hat. Unwichtig für die Bestimmung der Anlagepolitik ist hingegen der Wert, den Positionen zum Zeitpunkt $t$ haben, die den Wert des sonstigen Vermögens $v_{T}$ materiell begründen. Zwischen beiden Größen bestehen im allgemeinen Unterschiede. Leider wird in der Praxis gelegentlich die Gesamtbilanz auf die aktuellen Werte derjenigen Positionen abgestellt, die materiell das sonstige Vermögen begründen, nicht aber auf den Present Value von $v_{T}$. Wir zeigen also eine Unstimmigkeit mit dem in der Praxis üblichen Ansatz auf, und arbeiten die Konsequenzen für die Klärung der Ziele langfristiger Geldanlage aus.

In einer einfachen Betrachtung kann die Höhe des auf $T$ bezogenen sonstigen Vermögens als bekannt angenommen werden. Wir hatten es schon wie eine deterministische Größe ohne Tilde mit $v_{T}$ bezeichnet. Sicherheit und vollständige Information über $v_{T}$ vorausgesetzt, kann das sonstige Vermögen durch Diskontierung auf jeden vor $T$ liegenden Zeitpunkt bezogen werden. Dieser Present Value $P V$ ist

$$
P V\left(v_{T}\right)=v_{T} \cdot \exp \left(-(T-t) \cdot r_{f}\right),
$$

da $r_{f}$ der Zinssatz ist, die Rendite der risikofreien Anlage in stetiger Notation. ${ }^{16}$ Dieser PV spielt eine wesentliche Rolle bei der Bestimmung der Asset Allocation.

Die Person möchte durch die Asset Allocation $x_{t}$ über $0 \leq t \leq T$, mit der die Wahrscheinlichkeitsverteilung des Anlageergebnisses $\widetilde{w}_{T}$ beeinflußt wird, schließlich erreichen, dass der Erwartungsnutzen $E\left[u\left(\widetilde{w}_{T}+v_{T}\right)\right]$ maximiert wird.

16 Loewenstein/Thaler (1989) beobachten, dass Menschen nicht wie hier im Sinne finanzmathematischer Tradition exponentiell diskontieren, sondern zukünftige Zahlungen hyperbolisch diskontieren. 
Wir wissen vom Samuelson-Modell, dass dazu das totale Vermögen $\widetilde{w}_{t}+P V\left(z_{T}\right)$ zu allen Zeitpunkten $0 \leq t \leq T$ ein konstantes Exposure aufweisen soll, das lediglich von der durch $\gamma$ festgelegten RRA des Investors sowie von den Finanzmarktdaten $r_{f}, \mu$ und $\sigma$ abhängt (nicht aber von $T$ ). Dieses konstante Exposure des Gesamtvermögens sei für die folgenden Umformungen mit $X$ oder ausführlicher mit $X\left(\gamma ; r_{f}, \mu, \sigma\right)$ bezeichnet, zur Erinnerung: $X \equiv\left(\mu-r_{f}\right) /\left((1-\gamma) \cdot \sigma^{2}\right)$. Folglich verlangt die optimale Politik, wenn die Person zu $T$ sonstiges Vermögen $z_{T}$ haben wird, den in Aktien angelegten Geldbetrag $x_{t} \cdot w_{t}$ so zu wählen, dass er zu allen Zeitpunkten in der Relation $X$ zum Gesamtvermögen steht,

$$
X\left(\gamma ; r_{f}, \mu, \sigma\right)=\frac{x_{t} \cdot w_{t}}{w_{t}+P V\left(v_{T}\right)}
$$

Dies umgeformt und anders ausgedrückt muss stets der durch

$$
x_{t}=\frac{X \cdot\left(w_{t}+P V\left(v_{T}\right)\right)}{w_{t}}
$$

bestimmte Teil $x_{t}$ des Finanzvermögens in Aktien angelegt werden. ${ }^{17}$ Hat das Finanzvermögen einen kleinen Anteil am Gesamtvermögen (das sonstige Vermögen ist groß), ergeben sich entsprechend hohe Aktienquoten.

Beispiel: Ein Investor mit $R R A=10$, der stark risikoavers ist, bei den genannten Finanzmarktdaten $X=13,9 \%$ des Gesamtvermögens in Aktien halten. Der Investor möge zu $t=5$ ein Finanzvermögen von $w_{s}=300$ haben. $\mathrm{Zu} T=20$ soll er einem sonstigen Vermögen von $v_{20}=1000$ entgegen sehen. Diskontierung führt auf den auf $t=5$ bezogenen Barwert $P V(1000)=509\left(r_{f}=4,5 \%\right)$. Das Gesamtvermögen zu $t=5$ beträgt daher $w_{5}+P V\left(z_{20}\right)=300+509=809$. Von diesem Betrag möchte die Person $X=13,9 \%$ in Aktien halten. Sie möchte also Aktien für den Geldbetrag $809 \cdot 0,139=112$. Dazu muß sie für ihr derzeitiges

17 Weitere Nebenbedingungen kommen durch die deskriptive Entscheidungstheorie und andere Erkenntnisse hinzu. Nach Kahneman/Tversky (1979) entscheiden Menschen nicht aufgrund eines fernen, unsicheren Totalergebnisses, sondern aufgrund der Änderungen, die innerhalb einer kürzeren Evaluationsperiode eintreten. Hierbei zeigen sie eine Aversion gegen Verlust. Daher schränkt die kurzfristige Loss Aversion das möglich Exposure ein. Das maximal möglich Exposure hängt von der Länge der Evaluationsperiode ab, vergleiche Capon et. al. (1996). Wie Rechnungen des Autors zeigen, ergibt sich für eine Evaluationsperiode, die ein Jahr dauert, und realistische Finanzmarktdaten eine "gerade noch auszuhaltende" Aktienquote von ziemlich genau 50\%. 
Finanzvermögen, das 300 beträgt, die Aktienquote $x_{5}=112 / 300=37 \%$ wählen.

Besondere Aufmerksamkeit verdient die Behandlung einer Dynamik des sonstigen Vermögens. Bislang war $P V\left(v_{T}\right)$ als der auf den Zeitpunkt $t$ diskontierte Wert von $v_{T}$ verstanden, und das sonstige Vermögen $v_{T}$ zum Zeitpunkt $T$ sollte frühzeitig feststehen und bekannt sein. In der Realität ist es natürlich so, dass sich die unter dem Begriff "sonstiges Vermögen" zusammengefaßten Positionen - Humankapital, Pensionsansprüche, Immobilien und anderes mehr — in ihren Werten über die Zeit hinweg entfalten, entwickeln, und vielleicht sogar verfallen. Es gibt eine ausgeprägte, und gelegentlich modellierbare Dynamik. Beispielsweise ist der auf den Zeitpunkt $t$ bezogene Wert von Pensionsansprüchen nicht einfach der diskontierte Wert dieser Pensionsansprüche zum Zeitpunkt $T$, weil die Person zwischen $t$ und $T$ noch Beiträge einzahlt. Besonders stark dürfte die Dynamik beim Humankapital ausgeprägt sein, also bei der Fähigkeit, Arbeitseinkommen zu erzielen. Wenn der Zeitpunkt $T$ der "Fälligkeit" der Geldanlage gerade mit dem Eintritt in den beruflichen Ruhestand übereinstimmt, dann ist per definitionem das Humankapital (Barwert zukünftiger Arbeitseinkommen) gleich Null. Doch zuvor dürfte es Phasen geben, in denen das Humankapital sehr hoch ist. Schätzungen zeigen, dass für viele Menschen das Humankapital ein Maximum im Alter um 50 aufweist. ${ }^{18}$

Zwischen 0 und $T$ könnten es also zu verschiedensten Ereignissen kommen in dem Sinn, dass die Person neben ihrem Finanzvermögen über weitere wertvolle Positionen verfügt, die sich aufbauen und auch wieder abbauen und dabei einer eigenen Dynamik folgen. Der augenblickliche Wert zu $t$ dieser Positionen sei mit $v_{t}$ bezeichnet. Die Dynamik bedeutet, dass $v_{t}$ von $P V\left(z_{T}\right)$ für $t<T$ verschieden sein kann.

- Denn erfolgen noch weitere Investitionen in die entsprechenden Positionen (wie bei Pensionsansprüchen, die im Laufe der Jahre aufgebaut werden), gilt $v_{t}<P V\left(z_{T}\right)$.

- Kommt es zu einem Verfall dieser Positionen (wie das typischerweise beim Humankapital in der Altersphase zwischen 50 und 65 Jahren der Fall ist), gilt $v_{t}>P V\left(z_{T}\right)$.

Man wäre angesichts der Dynamik des sonstigen Vermögens, $v_{t} \neq P V\left(z_{T}\right)$, geneigt, die folgende Aussage zu treffen: "Zu jedem Zeitpunkt $t$ soll die Summe aus dem dann vorhandenem Finanzvermögen $w_{t}$ und dem augenblicklichen Wert dieser Positionen $v_{t}$ zum Anteil $X\left(\gamma ; r_{f}, \mu, \sigma\right)$ risikobehaftet angelegt sein (und nicht die Summe aus dem Finanzvermögen $w_{t}$ und dem Barwert $P V\left(z_{T}\right)$ des

18 Spremann (1995), Spremann/Winhart $(1997,1998)$. 
sonstigen Vermögens)." Viele Gesamtbilanzen in der Beratungspraxis setzen diese Formulierung um. Indessen ist dies nicht der korrekte Weg, denn Achtung: Die einzige Quelle für den "Nutzen" der Person ist das totale Vermögen zum Zeitpunkt $T$. Das totale Endvermögen ist das einzige Argument der Risikonutzenfunktion, und in diesem erscheint neben dem Anlageergebnis $\widetilde{w}_{T}$ nur noch der Wert $v_{T}$, den das sonstige Vermögen dann hat. Der auf den Zeitpunkt $t$ bezogene Wert dieses sonstigen Vermögens ist folglich $P V\left(v_{T}\right)=v_{T} \cdot \exp \left(-(T-t) \cdot r_{f}\right)$ und nicht $v_{t}$.

Ob Positionen, die "hinter dem sonstigen Vermögen stehen", zu $t$ einen anderen, vielleicht geringeren (Pensionsansprüche) oder höheren (Humankapital) Wert haben, ist in der gewählten Modellierung ohne Bedeutung. Beispiele:

- Eine Person legt im Alter von 50 Jahren ihren Geldbetrag $w_{0}=1000$ auf $T=15$ Jahre an, um im Alter der Pensionierung, mit 65 Jahren, "etwas zu haben". Bei der Bestimmung der Aktienquote soll neben $R R A=10$ oder $X=13,9 \%$ nur noch das Humankapital berücksichtigt werden. Im Alter von 50 Jahren $(t=0)$ steht die Person in der Blüte ihrer Arbeitskraft und schätzt ihr Humankapital auf $v_{0}=3000$. Indessen ist klar, dass zum Zeitpunkt $T=15$ der Pensionierung und der Fälligkeit der Geldanlage $v_{15}=0$ gilt. Deshalb gilt $P V\left(v_{15}\right)=0$ auch für alle Zeitpunkte zuvor. Die richtige Aktienquote für das Finanzvermögen $w_{0}=1000$ ist daher $x_{0}=13,9 \%$ und es werden für den Betrag $x_{0} \cdot w_{0}=139$ Aktien gekauft.

- Dieselbe Person ( $R R A=10, X=13,9 \%)$ legt im Alter von 35 Jahren den Geldbetrag $w_{0}=250$ auf $T=15$ Jahre an, im Alter von 50 Jahren "etwas zu haben". Bei der Bestimmung der Aktienquote soll das Humankapital berücksichtigt werden. Im Alter von 50 Jahren, bei Fälligkeit der Geldanlage $T=15$ steht die Person in der Blüte ihrer Arbeitskraft und schätzt, dass ihr Humankapital dann $v_{15}=3000$ betragen sollte. Ihr sonstiges Vermögen ist daher zum Zeitpunkt der Fälligkeit der Geldanlage recht hoch. Bezogen auf das heutige Alter von 35 Jahren $(t=0)$ ist der Present Value dieses sonstigen Vermögens $P V\left(v_{15}\right)=3000 \cdot \exp (-15 \cdot 0,045)=1527$. Das augenblickliche Gesamtvermögen der Person beträgt folglich $w_{0}+P V\left(z_{15}\right)=250+1527=1777$, und auf dieses Gesamtvermögen bezogen soll die Aktienquote $X=13,9 \%$ betragen, das heißt, es sollte der Geldbetrag $X \cdot\left(w_{0}+P V\left(z_{15}\right)\right)=0,139 \cdot 1777=247$ in Aktien angelegt werden. Von ihrem Finanzvermögen in Höhe $w_{0}=250$ nimmt die Person daher 247, um Aktien zu kaufen. Das entspricht einer Aktienquote von $x_{0}=247 / 250=99 \%$. Achtung: Auf die Höhe des Humankapitals zum Anlagezeitpunkt kommt es nicht an. 
Das sonstige Vermögen kann auch negativ sein. Samuelson hat, wie zuvor Rubinstein (1976), auf den Fall von Verbindlichkeiten hingewiesen. Jede Person muß, vor allem in einer Gesellschaft ohne soziale Hilfe, zumindest das Geld für das Existenzminimum (subsistence level) aufbringen. Diese Verbindlichkeit reduziert selbstverständlich das totale Vermögen. Vielleicht ist der dafür später zum Zeitpunkt $T$ benötigte Geldbetrag, wir bezeichnen ihn mit $z_{T}$, der Höhe nach schon frühzeitig bekannt. Die Pflicht, zu $T$ diesen Betrag zu haben, ist für die Person absolut bindend. Die Person wird deshalb schon zu Beginn der Geldanlage, zu $t=0$, den Barwert $z_{0}=z_{T} \cdot \exp \left(-r_{f} \cdot T\right)$ dieser auf die Seite legen und sicher anlegen. Nur der Rest steht für die bisher diskutierte Anlagestrategie zur Verfügung und ist neben dem sonstigen Vermögen das Argument der Risikonutzenfunktion.

\section{Die optimale Aktienquote}

Im Samuelson-Modell ist der Anlagehorizont $T$ eigentlich keine wichtige Größe, weil sich die optimale Aktienquote als vom Anlagehorizont unabhängig erweist. Der Anlagehorizont muß daher in der Beratung von Privatinvestoren nicht besonders thematisiert werden. Gibt es jedoch sonstiges Vermögen, wird der Anlagehorizont auf einmal bedeutend. Genauer gesagt kommt es nicht auf $T$ direkt an, sondern auf die Höhe $v_{T}$, die das sonstige Vermögen zu diesem Zeitpunkt haben wird.

Unterliegen Positionen wie Pensionsansprüche und Humankapital einer Dynamik, wird $v_{T}$ erheblich mit $T$ variieren. Folglich hängt die schließlich gewählte Aktienquote stark davon ab, ob die Person zum Zeitpunkt $T$, zu dem sie ihr Geld wieder haben möchte, "sonst noch etwas hat" oder eben nicht. Legt ein junger Mensch (ohne Pensionsansprüche) sein Geld auf das fünfzigste Lebensjahr an, kann die Aktienquote für das Finanzvermögen hoch sein, weil er ja ohnehin mit 50 noch sonst viel haben wird insofern als sein Humankapital dann hoch ist. Legt dieser junge Mensch (ohne Pensionsansprüche) sein Geld hingegen auf das fünfundsechzigste Lebensjahr an, muß die Aktienquote für das Finanzvermögen gering sein, weil er mit 65 nichts weiter hat, denn sein Humankapital ist dann gleich Null.

Die Wahl des Zeithorizonts hat daher einen großen Einfluß auf die Aktienquote, wenn es sonstiges Vermögen gibt und wenn dieses eine Dynamik im Sinne von $v_{t} \neq P V\left(z_{T}\right)$ aufweist. Wird der Horizont auf einen Zeitpunkt geringen sonstigen Vermögens gelegt, muß das Geld vorsichtig angelegt werden (weil bei Fälligkeit sonst nichts da ist). Wird der Horizont auf einen Zeitpunkt hohen sonstigen Vermögens gelegt, kann für das Geld eine höhere Aktienquote gewählt werden. In der Finanzberatung lautet die kritische Frage daher nicht unbedingt "wie hoch ist Ihre persönliche Risikoaversion". Von größerem Einfluß auf die Aktienquote des Fi- 
nanzvermögens ist die Antwort auf die Frage: "Was haben Sie denn sonst noch an Vermögen zu jenem Zeitpunkt, zu dem Sie Ihr Geld wieder haben wollen."

Diese Erkenntnis ist eine große Herausforderung für die Beratungspraxis. Wenn ein junger Mensch seine Vermögensanlage neu organisiert und eine Beratung aufsucht, wird immer die Frage gestellt, was das Ziel der Geldanlage sei und zu welchem Zeitpunkt das Anlageergebnis benötigt werde.

Wenn die Person antwortet, dass sie das Geld sogar vererben könnte, weil sie sonst noch genug habe und die Pension einmal ausreichen werde, empfiehlt sich aufgrund dieser Ausarbeitung des Samuelson-Modells eine hohe Aktienquote für das Finanzvermögen, denn der Present Value des sonstigen Vermögens ist aufgrund der finanziellen Situation des Kunden hoch, und der Anteil $X\left(\gamma ; r_{f}, \mu, \sigma\right)$

des Gesamtvermögens soll in Aktien angelegt werden. Das hat, auch bei geringerer Risikotoleranz, eine hohe Aktienquote für das Finanzvermögen zur Folge. Natürlich könnte die praktizierte Aktienquote nach oben beschränkt werden. Beispielsweise sprechen, bei einer Evaluationsperiode von einem Jahr, die in der Behavioral Finance beschriebenen Phänomene der Loss Aversion für eine Begrenzung der Aktienquote auf ziemlich genau 50\%, und es könnte beachtet werden, dass die Aktienquote, die den Modus maximiert, 46,3\% beträgt. Diese Argumente sprechen also dafür, in der betrachteten Situation, ungeachtet der genauen Risikoaversion des Investors (sofern sie nur nicht extrem hoch ist), das Finanzvermögen zur Hälfte in Aktien anzulegen oder eine leicht darunter liegende Aktienquote zu wählen.

Ganz anders ist die Situation, wenn die Person das "mit Rendite angelegte Geld" in einer Zeit benötigt, in der sie sonst nichts hat, oder in der sie das Anlageergebnis vielleicht benötigt (im Sinn eines negativen sonstigen Vermögens). In diesem Fall ist das totale Vermögen als Argument der Risikonutzenfunktion sogar kleiner als das Anlageergebnis. Die auf das Gesamtvermögen bezogene Aktienquote $X\left(\gamma ; r_{f}, \mu, \sigma\right)$ verlangt daher eine auf das Finanzvermögen bezogene Aktienquote $x_{t}$, die geringer als $X\left(\gamma ; r_{f}, \mu, \sigma\right)$ ist. Vereinfacht können wir sagen, dass dann — ungeachtet der Risikoaversion — das gesamte Finanzvermögen in Geldmarktinstrumenten und in Bonds anzulegen ist.

Um dieses Ergebnis der formalen Analyse anschaulich auszudrücken, lassen wir den Berater zum Privatinvestor sagen: "Ihre genaue Risikoaversion ist unwichtig, wichtig ist hingegen, ob sie ihre Geld einmal benötigen werden. Wenn Sie sich nur daran freuen, es zu haben, dann legen wir es zur Hälfte in Aktien an. Wenn Sie es jedoch irgendwann einmal benötigen — der Zeitpunkt ist unwichtig - legen wir das Geld sicher an: Es kommen nur Geldmarktinstrumente und Bonds in Frage"

Nur ein Nachsatz: Das sonstige Vermögen $v_{T}$ (beziehungsweise eine Verpflichtung oder ein negatives sonstiges Vermögen) wird zum Zeitpunkt der Planung der 
Geldanlage im Regelfall nicht wie bisher angenommen ein bekannter, feststehender Betrag sein. Es ist meistens unsicher, und in einigen Fällen dürfte es ein Exposure gegenüber dem Markt enthalten. Das ist beispielsweise der Fall, wenn neben den Aktien auch die Arbeitseinkommen der Person mit der allgemeinen Wirtschaftsentwicklung positiv korreliert sind. Die Unsicherheit ist durch die Schreibweise $\widetilde{v}_{T}$ auszudrücken, und anstelle der Diskontierung mit dem Zinssatz müßte der auf $t$ bezogene Wert von $\widetilde{v}_{T}$ durch Replikation bestimmt werden.

\section{Literatur}

Bamberg, Günter / Klaus Spremann (1981): Implications of Constant Risk Aversion, Zeitschrift für Operations Research, vol. 25, 205-224.

Blume, Marshall E. / Irwin Friend (1975): The Asset Structure of Individual Portfolios and Some Implications for Utility Functions, Journal of Finance 30, No. 2, 585-603.

Brennan, Michael J. (1998): The Role of Learning in Dynamic Portfolio Decisions, European Finance Review 1, 295-306.

Campbell, John Y. / Luis M. Viceira (2002): Strategic Asset Allocation — Portfolio Choice for Long-Term Investors, Oxford: University Press.

Noel Capon, Noel / Gavan J. Fitzsimons / Russ Alan Prince (1996): An Individual Lever Analysis of the Mutual Fund Investment Decision, Journal of Financial Services Reserach $10,59-82$.

Dréze, Jacques H. (1978): Axiomatic Theories of Choice, Cardinal Utility and Subjective Probability: a review, in Peter Diamond / Michael Rothschild (eds.): Uncertainty in Economics, New York: Acadic Press, 3-23.

Friend, Irwin / Marshall E. Blume (1975): The Demand for Risky Assets, American Economic Review, 900-922.

Friend, Irwin (1977): The Demand for Risky Assets: Some Extensions, in H. Levy, / M. Sarnat (Hrsg.): Financial Decision Making under Uncertainty, New York: Academic Press, 62-82.

Grossman, Sanford J. / Robert J. Shiller (1981): The Determinants of the Variability of Stock Market Prices, American Economic Review 71, 222-227.

Kahneman, Daniel / Amos Tversky (1979): Prospect Theory: An analysis of decisions under risk, Econometrica 47, 263-291.

Kränzlein, Klaus: Asset Allocation bei variablem Anlagehorizont, Bern: Verlag Haupt.

Luenberger, David G. (1979): Introduction to Dynamic Systems: Theory, Models, and Applications, New York.

Loewenstein, George F. / Drazen Prelec (1993): Preferences for Sequences of Outcomes, Psychological Review 100, No. 1, 91-108. 
Loewenstein, George F. / Richard H. Thaler (1989): Anomalies: Intertemporal Choice, Journal of Economic Perspectives 3, No. 4, 181-193.

Merton, Robert C. (1969): Lifetime Portfolio Selection under Uncertainty: The Continuous Time Case, in Merton, R. C. (1993): Continuous-Time Finance, Cambridge, MA: Blackwell Publishers, 97-111.

Merton, Robert C. (1971): Optimum Consumption and Portfolio Rules in a ContinuousTime Model, in Merton, R. C. (1993): Continuous-Time Finance, Cambridge, MA: Blackwell Publishers, 120-165.

Pindyck, Robert S. (1988): Risk Aversion and Determinants of Stock Market Behavior, Review of Economics and Statistics 70, 183-190.

Rubinstein, Mark (1976): The Strong Case for the Generalized Logarithmic Utility Model as the Premiew Model of Financial Markets, Journal of Finance 31, 551-571.

Samuelson, Paul A. (1969): Lifetime Portfolio Selection by Dynamic Stochastic Programming, Review of Economics and Statistics 51, 239-246.

Samuelson, Paul A. (1989): A Case at Last for Age-Phased Reduction in Equity, Proceedings of the National Academy of Science 86, 9048-9051.

Schneeweiß, Hans (1967): Entscheidungskriterien bei Risiko, Heidelberg: Springer.

Spremann, Klaus (1995): Asset Allocation im Lebenszyklus und Vintage-Programm, in B. Gehrig (Hrsg.), Private Banking, Zürich: Verlag NZZ, 115-146.

Spremann, Klaus / Stephanie Winhart (1997): Humankapital im Portfolio privater Investoren, Zeitschrift für Betriebswirtschaft 3, 145-167.

Spremann, Klaus / Stephanie Winhart (1998): Anlageberatung im Lebenszyklus, Finanzmarkt und Portfoliomanagement 12, 150-169.

Spremann, Klaus (2003), Portfoliomanagement, 2. Auflage, München: Oldenbourg Verlag, 367-370.

Telser, Lester G. (1955): Safety First and Hedging, Review of Economic Studies 23, 1-16.

Tobin, James (1958): Liquidity Preference as Behavior Towards Risk, Review of Economic Studies 25, 65-86.

Winhart, Stephanie (1999): Der Einfluss des Zeithorizonts auf die Asset Allocation — Ein Betrachtung unter besonderer Berücksichtigung des Investment Opportunity Set und der Risikoaversion, Bern: Verlag Haupt. 
